\title{
Temporal trends and geographic differences of insurance coverage for cancer drug trials in mainland China
}

\author{
Huiyao Huang ${ }^{1 *}$, Yu Tang ${ }^{1 *}$, Qi Fan ${ }^{1}$, Dawei Wu ${ }^{1}$, Jun Wang ${ }^{2}$, Shuhang Wang ${ }^{1}$, Hong Fang ${ }^{1}$, Yue Yu ${ }^{1}$, Yuan Fang ${ }^{1}$, Ying Bai ${ }^{1}$, \\ Chao Sun ${ }^{1}$, Anqi $\mathrm{Yu}^{1}, \mathrm{Zhu} \mathrm{Qi}^{3}$, Tao $\mathrm{Wei}^{3}$, Zhao Yan ${ }^{4}$, Ye Cao ${ }^{5}$, Min Jiang ${ }^{6}$, Yanfei Liu ${ }^{7}, \mathrm{Kunyan} \mathrm{Li}^{8}, \mathrm{Ning}^{1}{ }^{1}$
}

Cite this article: Huang HY, Tang Y, Fan Q, Wu DW et al: Temporal trends and geographic differences of insurance coverage for cancer drug trials in mainland China. Asia-Pac J Oncol 2020; 1: 45-51. https://doi.org/10.32948/ ajo.2020.10.11

\begin{abstract}
Background Data on insurance coverage of cancer drug trials in mainland China was of paucity, especially for its time trend and regional difference.

Methods Based on the national authoritative database, time trend analysis of insurance coverage of cancer drug trials was conducted, from both the perspectives of trials and participants. Meanwhile, group comparisons by region, drug type and study phase, were also performed. Mann-Kendall test was used for trend analysis, and chi-square test was used to conduct group comparisons.

Results A total of 1889 clinical trials were included, with 333 being international trials. In average, the insurance rate of trials was $79.8 \%$, and it was steadily increased by $6.8 \%$ annually from 2010 to 2019 . While the insurance coverage of Chinese participants was $67.6 \%$, and it showed a wavy upward trend. Compared with international trials $(91.9 \%)$, insurance rate of domestic trials $(77.2 \%)$ was significantly lower $(P<0.001)$, but the rate gap decreased gradually. The comparisons by region showed that, the insurance rate in Northeast China $(86.6 \%)$ was the highest, while that for Northwest China (40.0\%) was lowest. Additionally, traditional Chinese medicine trials, and BE studies had significantly lower rate of insurance.

Conclusion The insurance coverage of cancer drug trials in mainland China has been increasing steadily over the past decade. However, the regional differences are significant. To promote insurance coverage emphatically in underdeveloped areas could be our work emphasis. Although this study specially included cancer drug trials, the results could also provide reference for trials in all other fields.
\end{abstract}

Key words Neoplasms, clinical trial, insurance, time trend, regional difference

\footnotetext{
1. Department of Clinical Trial Center, National Cancer Center/National Clinical Research Center for Cancer/Cancer Hospital, Chinese Academy of Medical Sciences and Peking Union Medical College, Beijing, China.

2. National Center for Drug Evaluation, China Food and Drug Administration, Beijing, China.

3. School of Basic Medicine and Clinical Pharmacy, China Pharmaceutical University, Nanjing, China.

4. Department of Clinical Trial Center, Tianjin Medical University Cancer Institute \& Hospital, Tianjin, China.

5. Department of Clinical Trial Center, Sun Yat-sen University Cancer Center, Guangzhou, China.

6. Department of Clinical Trial Center, Peking University Cancer Hospital \& Institute, Beijing, China

7. Department of Clinical Trial Center, Fudan University Shanghai Cancer Center, Shanghai, China.

8. Department of Clinical Trial Center, Hunan Cancer Hospital, Changsha, China.

Correspondence: Ning Li (Director of Clinical Trial Office, National Cancer Center/National Clinical Research Center for Cancer/Cancer Hospital, Chinese Academy of Medical Sciences, Peking Union Medical College, 17 South Panjiayuan Lane, Chaoyang District, Beijing, 100021, People's Republic of China; E-mail: lining@cicams.ac.cn).

*These authors contributed equally to this work.
} 


\section{Introduction}

Rapid advancements in cancer treatment have critically put forward higher and faster requirements for clinical trial process, which is a series of mandatory and rigorous testing on investigational product, to evaluate its efficacy and safety before granting approval to the market [1]. With the great support from the national government, dramatic increasing trend regarding to clinical trials in China has been observed in mainland China [2], and China has become the world's second largest pharmaceutical consumer market [3]. According to the Statistics Report on National Adverse Drug Reactions, for the past decade, adverse drug reactions were increasing year by year in China (Figure 1), with the annual number of 1.49 million in 2017 [4].

Insurance is a key safeguard measure for the rights and interests of participants, as well as an important risk sharing mechanism for the sponsor. Meanwhile, it helps to avoid unnecessary disputes between participants with hospitals and ethics committees through by purchasing insurance. Since 2003, insurance for participants of clinical trials has been included in the national regulation in China, but it has not been protected by legislation $[1,5-6]$. Clinical trial claim disputes due to the absence of insurances happened sometimes in China. However, comprehensive analysis on insurance rate of clinical trials in China is of paucity. The few studies found related were mostly case studies, perspectives or original studies with small sample size [7-14]. Drug repositioning of existing drugs for novel indications is an attractive strategy as it maximizes the value of an existing drug by reducing costs and timelines involved in the development of new drugs. In recent years, repositioned drugs accounted for almost $30 \%$ of Food \& Drug Administration (FDA) newly approved drugs and vaccines $[6$, 7].

Based on the national authoritative database, "Platform for Registry and Publicity of Drug Clinical Trials" [15], this study was to analyze the time changes and regional differences of insurance rates for cancer clinical drug trials in China, so as to provide data support and advices for the improvement and implementation of relevant laws and regulations of clinical trial insurance and ethical review in mainland China.

\section{Materials and methods}

Data source: Platform for Registry and Publicity of Drug Clinical Trials

In 2013, the China Food and Drug Administration (CFDA) issued the notice that all the drug clinical trials approved by CFDA and conducted in China must be registered on the Platform for Registry and Publicity of Drug Clinical Trials (http://www.chinadrugtrials. org.cn/) before the first participant was enrolled, including bioequivalence (BE) studies, pharmacokinetics studies, phase I-IV drug trials [15].

Included public information can be divided into three parts, a) sponsor and registration items, such as sponsor and contact, date of first issue, etc.; b) basic management items of clinical trials, such as funding, leading unit, date of first ethical review, date of first enrollment, insurance, Data Monitoring Committee, etc.; c) scientific information on study design, such as indication, drug type (chemicals, biologic agent and others), inclusion and exclusion criteria, study phase, study scope, sample size, primary and secondary endpoints, etc.

\section{Data processing and key indicators}

From Jan. 2013 to Dec. 2019, a total of 8512 drug trials were registered on the "Platform for Registry and Publicity of Drug Clinical Trials". Data processing was mainly divided into the following three steps, screening for cancer clinical trials, followed by excluding trials initiated before Jan. 2010 according to date of first ethical review, and data reassignment. For detailed process, refer to Figure 2.

The main indicators of this study were insurance rate of clinical

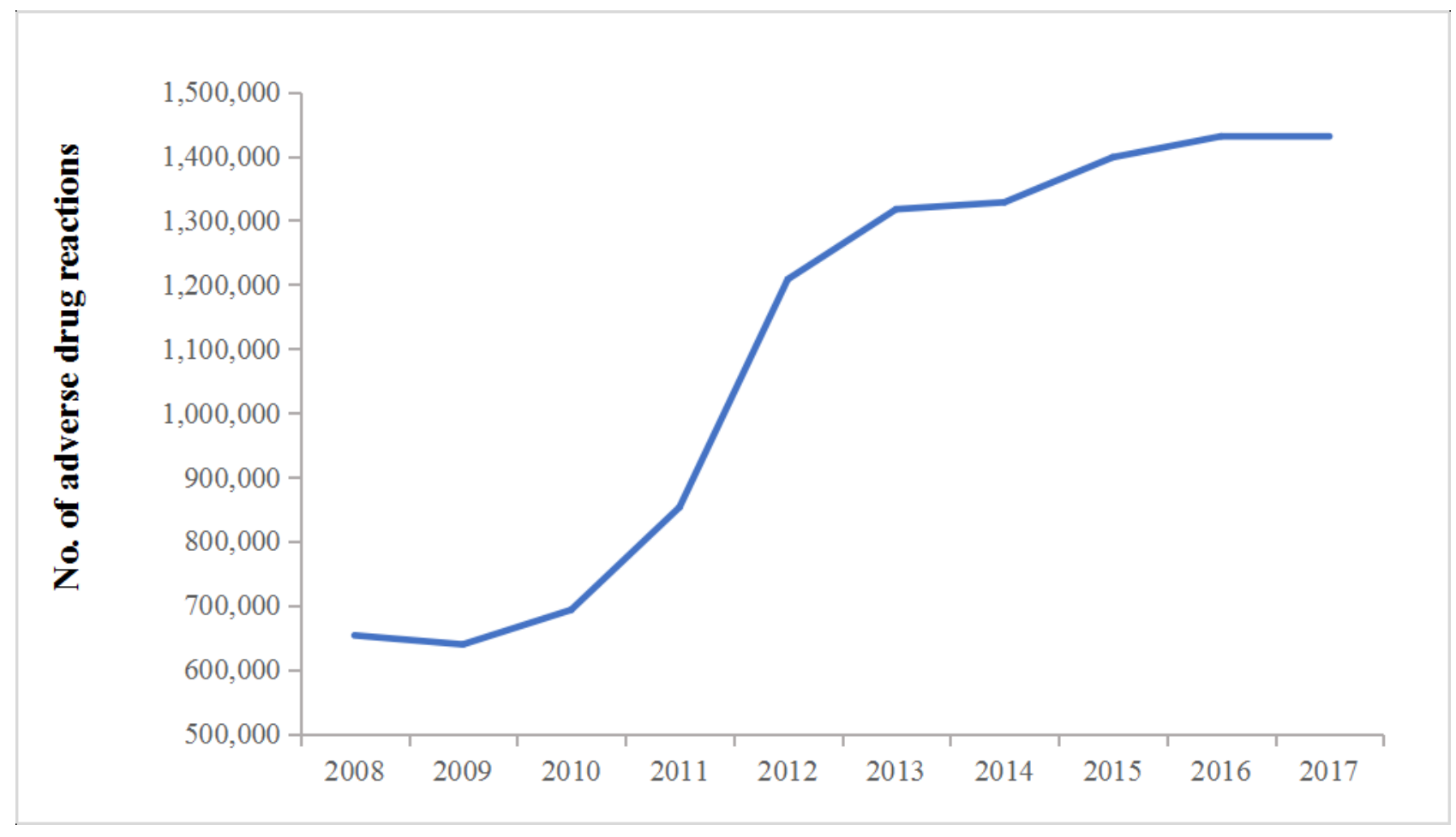

Figure 1. Annual adverse drug reactions in mainland China from 2008 to 2017. 


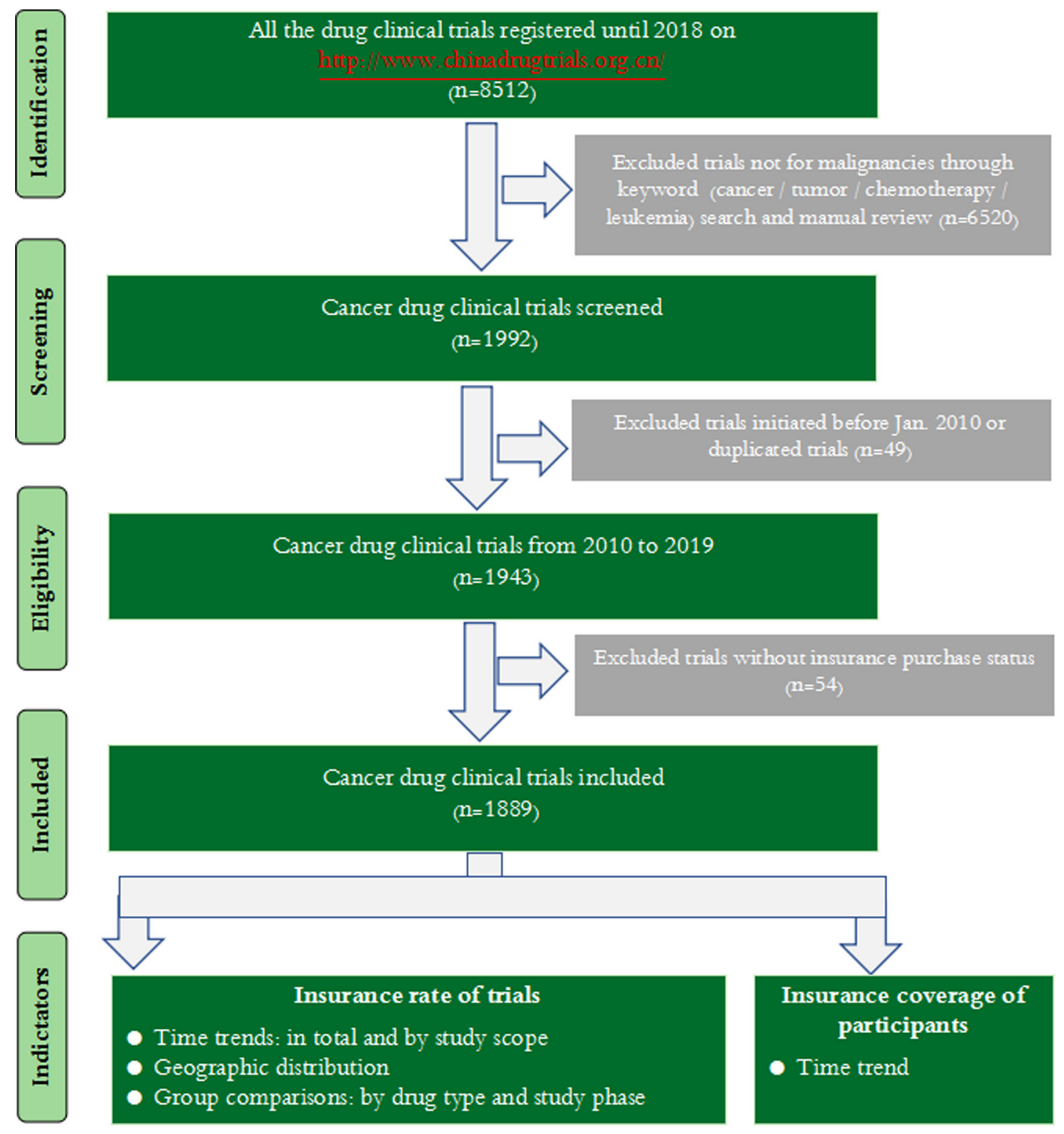

Figure 2. Flowchart of data processing.

trials and insurance coverage of participants. Both the temporal trends and subgroup analysis by study scope, drug type, study phase and region were conducted. In this study, time trend was calculated in line with the year of the first ethical review instead of the time of online submission or first enrollment, based on data availability and reliability. The geography of mainland China was divided into seven parts, including Northeast, North, East, South, Northwest, Southwest and Central China, based on the provinces where the leading unit of the trial was located.

\section{Statistical analysis}

SAS statistical software, version 9. 4 (SAS Institute, Cary/NC, US) was used for data processing and analysis. For descriptive analysis, no. (\%) was used for qualitative variables. The 10 -year time trends on insurance rate of trials in total and by study scope, as well as insurance coverage of participants were done using simple regression model. The average tempo was used to calculate an annual average increasing rate of above endpoints. Chi-square test was used for subgroup comparison of insurance rate by drug type, study phase and region. A two-tailed $p<0.05$ was considered statistically significant.

\section{Results}

Time trend on insurance rate of cancer drug trials

Cumulatively, a total of 1943 cancer clinical drug trials in China were registered on the platform over the last decade, with 1889 $(97.2 \%)$ trials reporting insurance purchase. Among the included 1889 trials, 1556 (82.4\%) were domestic while 333 (17.6\%) were international. Overall, the insurance rate of all the cancer drug clinical trials in China was $79.8 \%$, and that of international trials $(91.9 \%)$ was significantly higher than that of domestic trials $(77.2 \%)$ $(=36.5, \mathrm{P}<0.001)$. Overall, the insurance rate of drug trials in China has been steadily increasing from 2010 to 2019, with an average annual growth rate of $6.8 \%(\mathrm{~F}=88.7, \mathrm{P}<0.001)$. The average annual growth rate of insurance rate of domestic trials was 


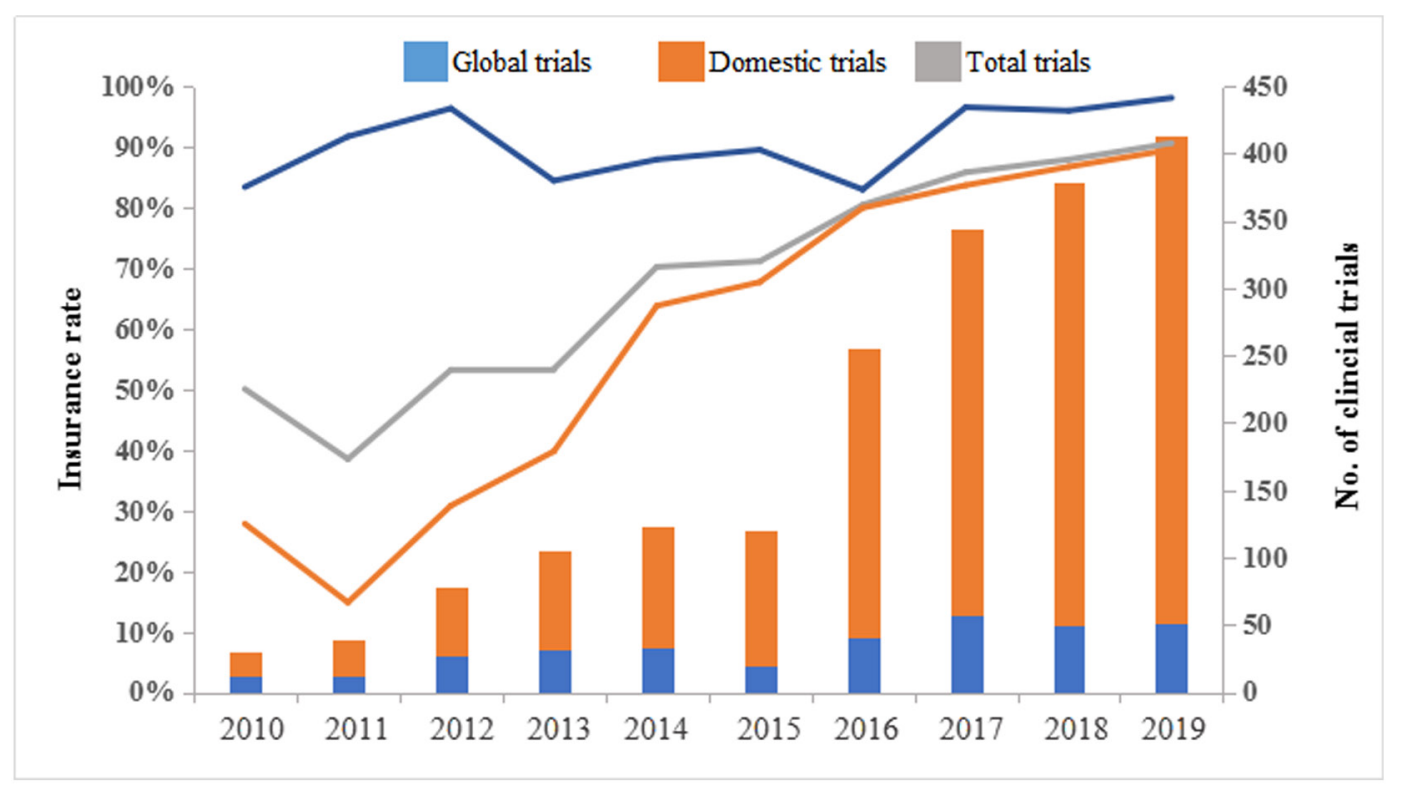

Figure 3. Trends on insurance rate of cancer drug trials by study scope in mainland China, 2010-2019.

$13.9 \%(\mathrm{~F}=87.3, \mathrm{P}<0.001)$, while that of international trials was relatively stable, and no significant change trend was found $(\mathrm{F}=$ $2.67, \mathrm{P}=0.141$, Figure 3 .

Time trend on insurance coverage of participants in cancer drug trials

Of the included 1889 trials, 1587 (84.0\%) had its sample size registered. In total, 255,950 participants were involved, with 233,156 (91.1\%) being Chinese. The insurance coverage of Chinese subjects was $67.6 \%$. For the past decade, the insurance coverage of Chinese subjects showed a wavy upward trend, which hit its low point in 2011 and 2012 that the coverage was less than $10.0 \%$. Since 2014, the growth trend has become relatively stable, and the coverage reached the highest $92.3 \%$ in 2019 . For more information, refer to Figure 4.

Insurance rates by different drug type and study phase

Drug type was classified into three kinds according to its molecular type, and there were 1179 (62.4\%) trials for chemicals, $689(36.5 \%)$ for biological agents, and 21 (1.1\%) for traditional Chinese medicine. The insurance rates of the above three kinds were $78.4 \%, 83.7 \%$ and $33.3 \%$, respectively, with significant

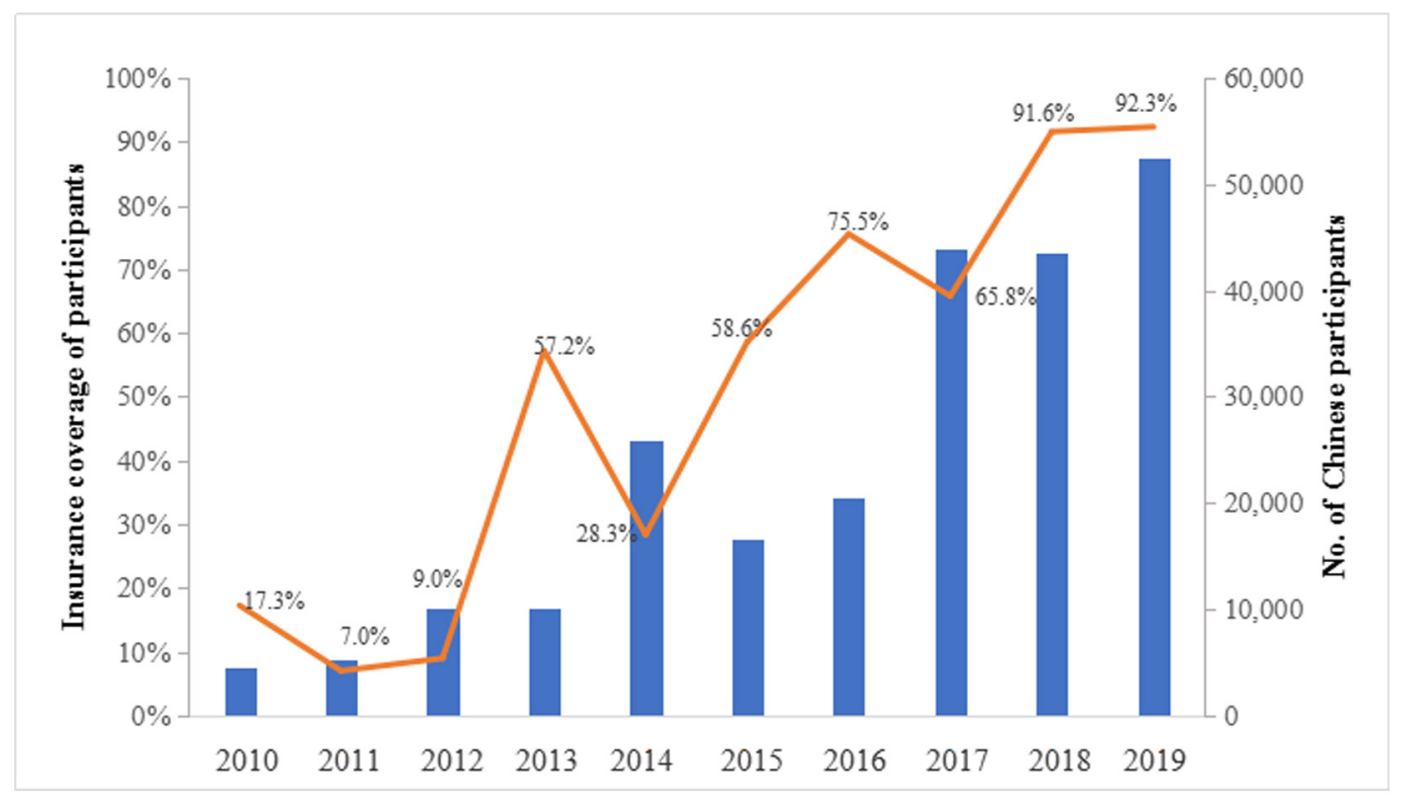

Figure 4. Trend on insurance coverage of participants in cancer drug trials in mainland China, 2010-2019. 
Table 1. Insurance coverage of participants in cancer drug trials in mainland China, by drug type and study phase.

\begin{tabular}{llll} 
Variables & No. of clinical trials & No. of trials with insurance & Insurance rate \\
\hline Total & 1889 & 1508 & $79.8 \%$ \\
Drug type & & & $78.4 \%$ \\
Chemicals & 1179 & 924 & $83.7 \%$ \\
Biologic agent & 689 & 577 & $33.3 \%$ \\
Other & 21 & 7 & \\
Study phasea & 827 & 675 & $81.6 \%$ \\
Phase I & 293 & 226 & $77.1 \%$ \\
Phase II & 477 & 421 & $88.3 \%$ \\
Phase III & 29 & 22 & $75.9 \%$ \\
Phase IV & 255 & 159 & $62.4 \%$ \\
BE & & & \\
\hline
\end{tabular}

The study phase of 8 trials were unclarified.

difference (chi-square $=36.3, \mathrm{P}<0.001)$. For different study phase, phase I trials accounted for the largest proportion (43.8\%), followed by phase III trials $(25.3 \%)$, phase II trials $(15.5 \%)$ and BE studies $(13.5 \%)$. The insurance coverage in BE studies was the lowest (62.4\%), followed by phase II trials (77.1\%), while it achieved the highest in phase III trials $(88.3 \%)$. For more information, refer to Table 1.

\section{Geographic difference of insurance rate of cancer drug trials}

The insurance rate displayed significant difference by geography (chi-square $=73.5, \mathrm{P}<0.001$ ). Therein, the rate (involved trials) for northeast China was the highest, amounting to $86.6 \%$, followed by $84.9 \%$ for south China, $82.1 \%$ for north China, $79.3 \%$ for east China, $61.2 \%$ for southwest China, $52.5 \%$ for central China, and the lowest $40.0 \%$ for northwest China. For the other 59 trials, they were international multicenter studies and didn't dominate leading units in China. More information was displayed in Figure 5.

\section{Discussion}

Based on national authoritative database, this longitudinal analysis pioneered to shed bright light on the overall trajectory and geographic distribution of insurance coverage for cancer drug clinical trials implemented in mainland China. The tremendous enrichment on changing law of trial insurance will provide essential support for the improvement and implementation of relevant laws and regulations in China, such as clinical trial insurance and ethical review.

The study found that both insurance rate and coverage of cancer drug trials in China showed increasing trends, especially for domestic clinical trials with an average growth rate of $13.9 \%$, which was consistent with the results of previous smallscale studies [7, 11]. There are several reasons, including the gradual improvement of the awareness of the public, sponsors and researchers to protect rights and interests, the concomitant appearance of clinical trial related insurance, as well as guidance of medical regulatory policies and implementation of ethical review. Since 2003, a series of regulations and guidance were issued to impose requirements on sponsors to provide coverage for individuals participating in clinical trials, and to explicitly require ethic committee to review insurance documents, such as "Good Clinical Practice", "Guidelines for the Ethical Review of Drug Clinical Trials" and "Methods for Ethical Review of Biomedical Research Involving People" [1, 5-6].

Geographic differences of insurance rate for cancer drug trials implemented in mainland China were observed. On the one hand, compared with international trials, the overall insurance rate of domestic trials is relatively low, especially for trials initiated before 2016 that the gap of insurance rate between international and domestic trials was more than $20 \%$. This is largely correlated with the lack of legislative protection, the consciousness deficiency of rights safeguarding, as well as the inadequate development of commercial insurance. President Obama implemented the Affordable Care Act (ACA) of 2010, which resulted dramatic changes for health care system in United States, and the increase of insurance coverage was gradually displayed. Worth noting, 71\% of the participants was insured by commercial insurance [16].

On the other hand, the insurance rate among the seven regions in mainland China was also uneven, that the insurance rates in northwest, central and southwest China were significantly lower, which is consistent with the unbalanced distribution of medical resources and developmental level in China. This could be also related to the unbalanced development of ethics committees and insurance companies. Therefore, to improve the insurance rate for trial participants in underdeveloped regions could be one emphasis and direction for ethics committee and related stakeholders in the future.

Meanwhile, the insurance rate of traditional Chinese medicine is significantly lower than that of chemicals and biological products. As indeed, the incidence of adverse reactions in clinical trials on traditional Chinese medicine is relatively low, and the awareness 


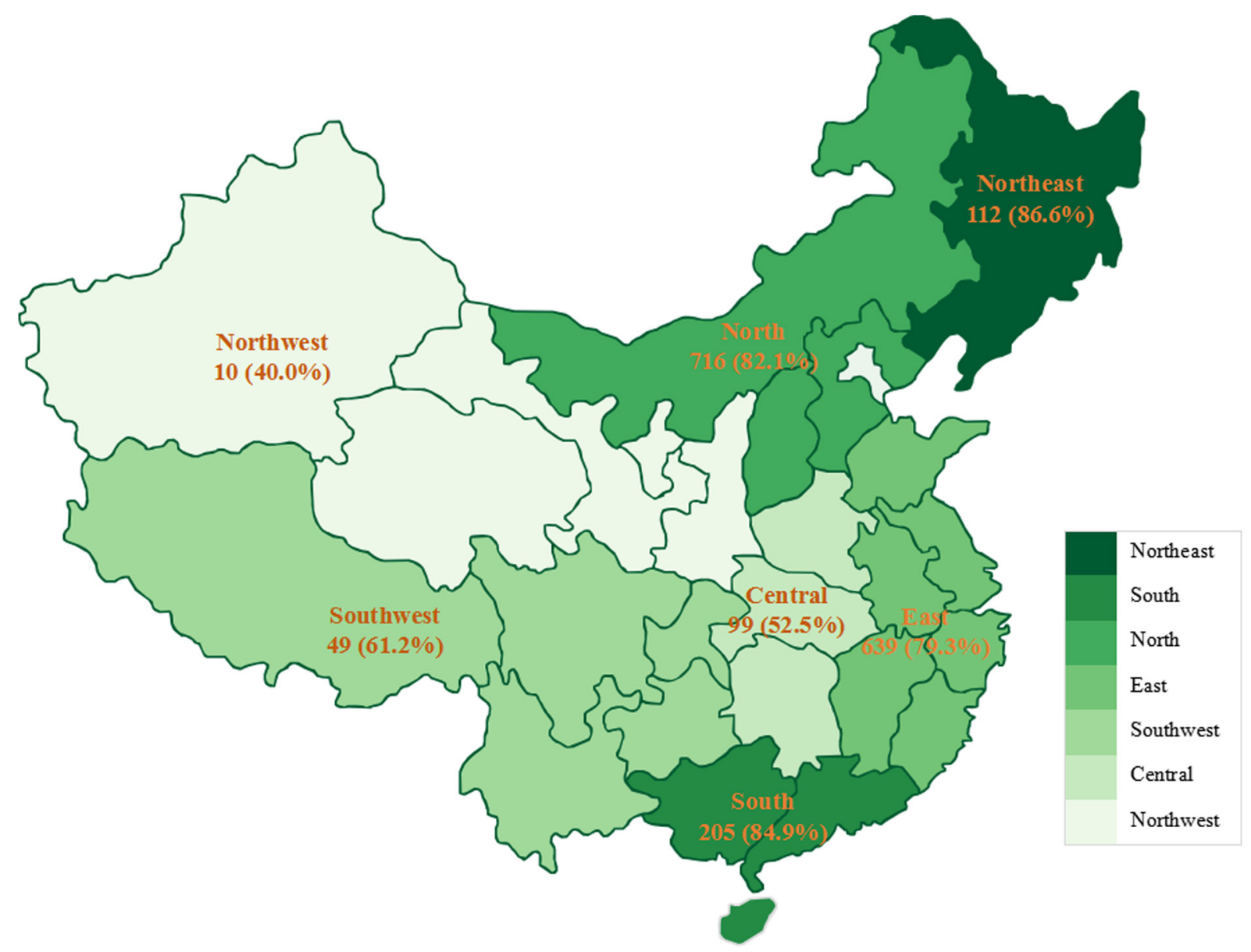

Figure 5. Insurance rate of cancer drug trials in mainland China, by geography.

of related trial stakeholders could be therefore weaker. In addition, from the perspective of governmental regulation, in order to promote the development of traditional Chinese medicine, the supervision of clinical trials on traditional Chinese medicine was postponed [17]. Even, it's suggested that traditional Chinese medicine that meet relevant requirements can be exempted from application for efficacy study and clinical trial study [18]. With the overall improvement of the rights protection awareness, security risks and medical disputes caused by direct use of drugs without clinical trials proven should be paid more and more attention.

Our study also has some limitations. The database may not cover registered clinical trials completed before 2013, though the registration is mandatory and supplementary registration was required for ongoing clinical trials in 2013. To keep consistent with previous published article, the initial year of clinical trials in this study are marked by the time of first ethic committee approval instead of the time of online submission or first enrollment [2]. In addition, this study had special focus on the insurance of registered clinical trials, further evidence on non-registered clinical trials was still needed, including trials initiated by researchers on new technologies, new methods or expanded indications.

Over the last decade, the insurance rate and coverage of cancer drug trials in mainland China has been increasing steadily year by year, reaching $90.6 \%$ in 2019 . To pertinently improve the insurance rate for trial participants in underdeveloped regions could be one emphasis and direction for ethics committee and related stakeholders in the future. Meanwhile, to establish multi-center cooperation platform and to conduct comprehensive survey on the insurance situation of non-registered clinical trial initiated by researchers could also be an important direction of future efforts.

\section{Ethics approval and consent to participate}

The Ethics Committee of National Cancer Center, Cancer Hospital, Chinese Academy of Medical Sciences and Peking Union Medical College provided the ethical approval for this study. (Ref: 18-028/1629) Informed consent exemption was approved as this study was performed based on registered trial-level data instead of 
individual-level data.

\section{Funding}

The analysis and interpretation of the manuscript was supported by "CAMS Innovation Fund for Medical Sciences (CIFMS)" and "Platform Improvement of Clinical Trial Capability" (2020-I2M-2 007).

\section{Author contributions}

HYH and YT contributed to framework planning and draft writing, as well as data quality control, analysis and interpretation. NL led the overall framework planning and data interpretation. DWW, QF and JW participated in data quality control and data interpretation. SHW, HF, YY, YB, YF, CS, AQY, DDC, QZ and WT provided administrative, technical and material support. ZY, YC, MJ, YFL and KYL participated in framework planning and contributed to data interpretation. All the authors reviewed and revised the manuscript. All the authors reviewed and revised the manuscript.

\section{Competing interests}

All authors disclose no competing interests.

\section{References}

1. China Food and Drug Administration. Good Clinical Practice, http://samr.cfda.gov.cn/WS01/CL1031/24473. html (2003, accessed 10 March 2020).

2. Li N, Huang HY, Wu DW, et al. Changes in clinical trials in mainland China over the decade 2009-18: a systematic review. Lancet Oncol. 2019; 20(11): 619-626. Doi: 10.1016/S1470-2045(19)30491-7.

3. IMS Institute for Healthcare Informatics. Outlook for Global Medicines through 2021, https://www.iqvia.com/en/newsroom/2016/ quintilesims-institute-forecast (2016, accessed 10 March 2019).

4. China Food and Drug Administration. Annual Report for National Adverse Drug Reaction Monitoring, http://www.cdr-adr.org.cn/ xwdt/201804/ t20180419_20011.html (2018, accessed 10 March 2020).

5. China Food and Drug Administration. Guidelines for the Ethical Review of Drug Clinical Trials, http://www.gov.cn/gzdt/2010-11/08/ content_1740976. htm (2010, accessed 10 March 2020).

6. National Health and Family Planning Commission. Methods for Ethical Review of Biomedical Research Involving People, http:// www.gov.cn/gongbao/content/2017/content_5227817. htm (2016, accessed 10 March 2020).

7. Wang J, Wen K, Cao J, et al. Status quo of risk management of drug clinical trials in China from the insurance situation of drug clinical trials in our hospital. Chin J New Drugs Clin Rem 2013; 32(12): 946949.

8. Shan AL, Liang Y and Cui YM. Investigation and analysis of providing insurance for subjects in clinical trials of new drugs and medical devices. Chin J Clin Pharmacol 2013; 29(7): 483-487.

9. Yang Z, Li CX and Wu ZA. Discussion on strengthening the protection of subjects' rights and interests in clinical trials. China Pharm 2013; 16(4): 610-613.

10. Liang YF. Thoughts on legal protection of subjects in drug clinical trials. Sci Techn Innov Herald 2014; 11(18): 223-224.

11. Wang CQ. Current situation and countermeasures of clinical trial insurance Jinan Univ, 2014.

12. Huang D, Li B, Weng H, et al. Pregnancy, insurance purchase, protocol violation and integrity of inspectors in clinical studies. Chin J Evid-based Cardiovasc Med 2017; 9(8): 897-899.

13. Sun HS and Zhang XL. Analysis and reflection on the current situation of drug clinical trial insurance in China. Chin J New Drugs Clin Rem 2016; 35(01): 27-31.

14. Wen K, Wang J and Wang R. "Compulsory option" of new drug clinical trial insurance. Chin J New Drugs Clin Rem 2014; 33(1): 1921.

15. China Food and Drug Administration. Platform for Registry and Publicity of Drug Clinical Trials, http://www.chinadrugtrials.org.cn/ (accessed 20 March 2020).

16. Mackay CB, Gurley-Calvez T, Erickson KD and Jensen RA. Clinical trial insurance coverage for cancer patients under the Affordable Care Act. Contemp Clin Trials Commun 2015; 17(2): 69-74. DOI: 10.1016/j.conctc.2015.12.002.

17. Cyranoski D. China to roll back regulations for traditional medicine despite safety concerns. Nature 2017; 551(7682): 552-553.

18. China Food and Drug Administration. Simplified Registration Approval Regulation for Classic Prescription and Compound Preparation of Traditional Chinese Medicine (draft for comments), http://samr.cfda.gov.cn/WS01/CL0778/178324.html (2017, accessed 21 June 2019). 